\title{
A descriptive analysis of a representative sample of pediatric randomized controlled trials published in 2007
}

\author{
Michele P Hamm¹, Lisa Hartling ${ }^{1 *}$, Andrea Milne', Lisa Tjosvold', Ben Vandermeer ${ }^{1}$, Denise Thomson',
} Sarah Curtis ${ }^{1,2,3}$, Terry P Klassen ${ }^{4}$

\begin{abstract}
Background: Randomized controlled trials (RCTs) are the gold standard for trials assessing the effects of therapeutic interventions; therefore it is important to understand how they are conducted. Our objectives were to provide an overview of a representative sample of pediatric RCTs published in 2007 and assess the validity of their results.

Methods: We searched Cochrane Central Register of Controlled Trials using a pediatric filter and randomly selected 300 RCTs published in 2007. We extracted data on trial characteristics; outcomes; methodological quality; reporting; and registration and protocol characteristics. Trial registration and protocol availability were determined for each study based on the publication, an Internet search and an author survey.

Results: Most studies (83\%) were efficacy trials, 40\% evaluated drugs, and 30\% were placebo-controlled. Primary outcomes were specified in 41\%; $43 \%$ reported on adverse events. At least one statistically significant outcome was reported in $77 \%$ of trials; $63 \%$ favored the treatment group. Trial registration was declared in $12 \%$ of publications and $23 \%$ were found through an Internet search. Risk of bias (ROB) was high in 59\% of trials, unclear in 33\%, and low in $8 \%$. Registered trials were more likely to have low ROB than non-registered trials (16\% vs. 5\%; $p=0.008)$. Effect sizes tended to be larger for trials at high vs. low ROB $(0.28,95 \% \mathrm{Cl} 0.21,0.35$ vs. $0.16,95 \% \mathrm{Cl} 0.07,0.25)$. Among survey respondents (50\% response rate), the most common reason for trial registration was a publication requirement and for non-registration, a lack of familiarity with the process.

Conclusions: More than half of this random sample of pediatric RCTs published in 2007 was at high ROB and three quarters of trials were not registered. There is an urgent need to improve the design, conduct, and reporting of child health research.
\end{abstract}

\section{Background}

Randomized controlled trials (RCTs) are considered the gold standard for research on therapeutic interventions and provide the best evidence to inform and guide clinical decision-making. Currently the number of pediatric trials conducted and published lags behind that for adults $[1,2]$. In addition, little is known about the risk of bias, or validity, of pediatric RCTs.

\footnotetext{
* Correspondence: hartling@ualberta.ca

1 Alberta Research Centre for Health Evidence, Department of Pediatrics,

University of Alberta. Edmonton, Canada

Full list of author information is available at the end of the article
}

Substantial evidence demonstrates that particular study design features increase the likelihood of systematic error, or bias, most often resulting in over-estimation of treatment effects. Risk of bias (ROB) reflects the degree to which the results of a trial should be believed [3]. Building on previous research around methodological quality of RCTs $[4,5]$, the Cochrane Collaboration recently introduced a tool designed to appraise $\mathrm{ROB}$, encompassing six domains related to the internal validity of a trial: sequence generation, allocation concealment, blinding, incomplete outcome data, selective outcome reporting, and "other" potential threats to validity [3]. 
Recent initiatives to address some of the biases associated with the design, conduct, and reporting of trials include the International Committee of Medical Journal Editors' (ICMJE) statement on trial registration [6] and reporting guidelines (http://www.equator-network.org) such as the CONSORT Statement (Consolidated Standards of Reporting Trials) [7]. Trial registration is integral in addressing the bias associated with selective outcome reporting by ensuring that investigators prospectively provide details on their trial, allowing for increased transparency and accountability [8]. The CONSORT Statement was developed to ensure adequate and transparent reporting upon completion of the trial and comprises a checklist of items that should be included in the publication of any RCT. Evidence suggests that these strategies have positively influenced the quality of published trials [9-11], but this has yet to be assessed in pediatrics.

Given these recent initiatives to improve reporting and assess $\mathrm{ROB}$, we aimed to describe the state of pediatric evidence using a representative sample of child health RCTs published in 2007. Specific objectives were to examine: 1) methodological quality, including ROB, and its association with effect estimates; 2) the rate of trial registration and author reasons for registration and nonregistration; and, 3) availability of trial protocols and their consistency with publications.

\section{Methods}

\section{Sample Selection}

Using a pediatric filter, the Cochrane Central Register of Controlled Trials (CENTRAL) was searched for trials published in 2007 [12]. CENTRAL is comprised of records of studies indexed in Medline and Embase, as well as hand-search results, grey literature, and the trials registers of Cochrane Review Groups [13]. As such, this provided a thorough search for pediatric controlled trials. Two thousand eight hundred thirty-two trials were randomly ordered using a computer-generated list, were screened consecutively for relevance, and the first 300 (approximately 10\%) RCTs matching the criteria below were selected. Trials were included if they were published in English and included participants aged 0 to 18 years. If a trial studied both children and adults, it was included if the upper age limit was $\leq 21$ years [13].

\section{Data Extraction}

Data were extracted on: publication (e.g., type of journal, impact factor) and trial characteristics; outcomes and conclusions; methodological quality and reporting; and trial registration and protocol characteristics related to outcomes. Data extraction was completed by one reviewer with an independent second review on a randomly selected $10 \%$ sample. Discrepancies were resolved through consensus and were negligible. Trial registration and protocol availability were determined for each study based on publication details, an Internet search, and author follow-up.

\section{Assessment of Methodological Quality and Reporting}

Given the range of quality assessment methods available, and the widespread use of many, methodological quality and reporting were assessed using multiple tools: the Jadad scale [4] and allocation concealment [5], as well as the Cochrane ROB tool [3] and the 2001 CONSORT Statement [14]. The Jadad scale is a five-point scale based on the description of randomization, doubleblinding, and withdrawals or losses to follow-up; a score of 5 indicates highest quality. Allocation concealment was assessed as adequate, inadequate, or unclear. Nearly all trials in our sample were efficacy trials; therefore we focused on the original CONSORT Statement. The 2001 CONSORT checklist was the most recently published version at the time of data extraction, and assesses reporting with respect to 22 items. Each item was assessed as fully, partially, or not met.

The ROB tool was applied based on guidelines established by The Cochrane Collaboration [3], with some modifications specific to our investigation (see Additional file 1). These consist of decision rules that have been developed by our centre that have been used in conjunction with the Cochrane guidelines to increase consistency across reviewers. An overall assessment of ROB was made as high, low, or unclear based on the criteria from the Cochrane handbook: if any of the six domains were judged to be at high risk of bias, the overall risk was considered high; if any were judged to be at unclear risk of bias and none at high risk, the overall risk was unclear; and if all six domains were judged to be at low risk of bias, the overall risk was low. The tool was pilot tested by all members of the study team. Trials were assessed independently by two trained reviewers who arrived at consensus for each of the six items.

\section{Trial Registration and Protocol Availability}

To determine whether or not trials were registered, details were first sought in the publication. If a declaration was not made, we searched through the International Clinical Trials Registry Platform (ICTRP) search portal maintained by the World Health Organization (WHO). If not found, the following registries were searched in order: ClinicalStudyResults.org, Memorial Sloan-Kettering Cancer Center, Current Controlled Trials Meta-Register, and CenterWatch. While there was some overlap in registries searched (i.e. ISRCTN.org is included in both the ICTRP portal and the Current Controlled Trials Meta-Register), each register contained unique databases. If a trial was not found in any of these 
registries, we conducted a Google search using the names of the first, last, and/or corresponding authors and key words. When available, data from the registry or from protocols found in our search were compared to the publication.

A 28-question survey regarding trial registration and protocol availability was sent to all corresponding authors with current email contact information ( $\mathrm{n}=$ 290; see Additional file 2). The initial invitation and survey link was followed by two reminders containing the same information. Protocols were requested from authors. Ethical approval was obtained from the Health Research Ethics Board at the University of Alberta prior to survey implementation.

\section{Analysis}

Data were analyzed descriptively, using means and standard deviations or medians and ranges for continuous variables and proportions for categorical variables. Effect sizes were computed for 236 trials with sufficient data based on the primary outcome for that trial. The effect size was a standardized mean difference when the primary outcome was continuous and a converted odds ratio when dichotomous [15]. Effect sizes were pooled using DerSimonian-Laird random effects for each of the three ROB categories (high, low, unclear). To compare ROB for certain covariates, a reference category was chosen within each variable classification and odds ratios comparing the number of high/unclear risk trials to low risk trials were computed with $95 \%$ confidence intervals.

\section{Results}

\section{Description of Study Sample}

Publication and trial characteristics of our sample of 300 trials are shown in Table 1. The majority of trials used parallel designs (89.7\%), were efficacy trials (82.7\%), and were published in specialty journals (78.6\%). Evaluation of pharmacological interventions was most common $(40.3 \%)$ and $30 \%$ of trials were placebo-controlled. While all major geographic areas were represented, the majority of authors were from Europe $(40.3 \%)$ and North America (29.0\%). Each study was categorized using the review groups of The Cochrane Collaboration: neonatal (9.3\%), oral health (7.7\%), and developmental, psychosocial, and learning problems (6.7\%) were most represented.

\section{Methodological Quality}

The median Jadad score was 2 (IQR 2-3). Allocation concealment was adequate in $21.7 \%$ of trials, while $75.7 \%$ were unclear (Table 2 ). Only three trials $(1.0 \%)$ sufficiently addressed all 22 items of the CONSORT

Table 1 Publication and trial characteristics $(\mathrm{N}=300)$

\begin{tabular}{ll}
\hline Study Characteristic & N (\%) \\
\hline Continent of corresponding author & $11(3.7)$ \\
Africa & $58(19.3)$ \\
Asia & $16(5.3)$ \\
Australia & $91(30.3)$ \\
Europe (excluding UK) & $87(29.0)$ \\
North America & $7(2.3)$ \\
South America & $30(10.0)$ \\
United Kingdom &
\end{tabular}

Type of journal

General medical journal $\quad 19$ (6.3)

Specialty medical journal $\quad 166(55.3)$

General pediatric journal $\quad 45$ (15.0)

Specialty pediatric journal $\quad 70(23.3)$

\begin{tabular}{ll}
\hline Study design & \\
RCT parallel & $269(89.7)$ \\
RCT crossover & $19(6.3)$ \\
RCT factorial & $5(1.7)$ \\
Other & $7(2.3)$ \\
\hline Study type & \\
Efficacy/Superiority & $248(82.7)$ \\
Equivalence & $9(3.0)$ \\
Non-inferiority & $13(4.3)$ \\
Not declared & $2(0.7)$ \\
None of the above & $25(8.3)$ \\
Unclear & $3(1.0)$
\end{tabular}

Nature of intervention

\begin{tabular}{ll} 
Drug & $121(40.3)$ \\
Vaccine & $16(5.3)$ \\
Natural health product & $26(8.7)$ \\
Device & $44(14.7)$ \\
Other & $93(31.0)$ \\
\hline Placebo-controlled & $90(30.0)$ \\
\hline Number of centres & \\
Multicentre & $105(35.0)$ \\
Single Centre & $179(59.7)$ \\
Unclear & $16(5.3)$
\end{tabular}

\begin{tabular}{ll}
\hline Sample size & $785.2(5837.3)$ \\
Mean (SD) & $83(6-71,799), 10$ - \\
Median (range), IQR & 7079 \\
\hline Data Monitoring Committee established & $14(4.7)$ \\
\hline Any adverse events reported & $129(43.0)$ \\
\hline Funding source & $194(64.7)$ \\
Declared & $67 / 194(34.5)$ \\
Industry Sponsored & $123(41.0)$ \\
\hline Primary outcome explicitly reported & $230(76.7)$ \\
\hline At least one statistically significant outcome & \\
\hline Intervention favored & $189(63.0)$ \\
Treatment & $19(6.3)$ \\
Control &
\end{tabular}


Table 1 Publication and trial characteristics $(\mathrm{N}=300)$ (Continued)

\begin{tabular}{ll}
\hline Neither & $92(30.7)$ \\
\hline Common primary diagnostic categories & $17(5.7)$ \\
Acute Respiratory Infections & $14(4.7)$ \\
Airways & $18(6.0)$ \\
Anaesthesia & $20(6.7)$ \\
Developmental, Psychosocial, and Learning & \\
Problems & $10(3.3)$ \\
Ear, Nose, and Throat Disorders & $19(6.3)$ \\
Infectious Disease & $15(5.0)$ \\
Metabolic and Endocrine Disorders & $28(9.3)$ \\
Neonatal & $23(7.7)$ \\
Oral Health & $16(5.3)$ \\
Public Health &
\end{tabular}

Statement (IQR 13-17) with another eight (2.7\%) at least partially meeting all requirements (IQR 15-19). The remaining 289 trials (96.3\%) failed to report at least one, and up to 14 recommended items. Overall, the median number of items that were adequately addressed was 15 , and five for those partially addressed. Descriptions of the "method used to implement the randomization sequence" (item 9) and "who generated the allocation sequence and enrolled and assigned participants" (item 10) were the most under-reported, with 214 (71.3\%) and 229 (76.3\%) trials not meeting these criteria respectively.

Overall ROB was low for 23 trials (7.7\%), unclear for 99 (33.0\%), and high for 178 (59.3\%) (Table 2). Much of the uncertainty in rating studies was due to unclear

Table 2 Assessments of methodological quality $(N=300)$

\begin{tabular}{ll}
\hline Methodological Quality Indicator & N (\%) \\
\hline Jadad & $2.6(1.2)$ \\
Mean (SD) & $2(0-5)$ \\
Median (range) & $65(21.7)$ \\
\hline Allocation Concealment & $227(75.7)$ \\
Adequate & $8(2.7)$ \\
Unclear & \\
Inadequate & $23(7.7)$ \\
\hline Risk of Bias & $99(33.0)$ \\
Low & $178(59.3)$ \\
Unclear & \\
High & $15(4-22)$ \\
\hline CONSORT Statement & $5(0-14)$ \\
Items fully addressed (median, range) & $2(0-7)$ \\
Items partially addressed (median, range) & $2 / 290(0.7)$ \\
Items not addressed (median, range) & $37(12.3)$ \\
\hline Trial registered & $69(23.0)$ \\
Declared in publication & \\
Registration found online & \\
\hline Study protocol available from corresponding author & \\
\hline
\end{tabular}

Table 3 Risk of bias assessments by domain $(\mathrm{N}=300)$

\begin{tabular}{llll}
\hline Domain & \multicolumn{3}{l}{ Risk of bias assessments - N (\%) } \\
\hline & High & Unclear & Low \\
\hline Sequence generation & $8(2.7 \%)$ & $143(47.7 \%)$ & $149(49.7 \%)$ \\
\hline Allocation concealment & $8(2.7 \%)$ & $217(72.3 \%)$ & $75(25.0 \%)$ \\
\hline Blinding & $41(13.7 \%)$ & $108(36.0 \%)$ & $151(50.3 \%)$ \\
\hline Incomplete data & $60(20.0 \%)$ & $53(17.7 \%)$ & $187(62.3 \%)$ \\
\hline Selective reporting & $48(16.0 \%)$ & $6(2.0 \%)$ & $246(82.0 \%)$ \\
\hline "Other" sources of bias & $85(28.3 \%)$ & $109(36.3 \%)$ & $106(35.3 \%)$ \\
\hline
\end{tabular}

reporting. Selective outcome reporting was rated as low ROB in nearly all trials. "Other" sources of bias included inappropriate influence of the study sponsor (e.g. industry funding without separation from the conduct of the trial), imbalance in baseline characteristics, and designspecific issues (e.g., factors related to cluster RCTs or cross-over trials), and was the domain that was least often addressed satisfactorily (Table 3 ). Trials at low ROB had higher mean Jadad scores and were more likely to report adequate means of allocation concealment than those at high ROB (Table 4).

Effect sizes tended to increase from studies at low $(0.16,95 \%$ CI $0.07,0.25)$ to high $\operatorname{ROB}(0.28,95 \% \mathrm{CI}$ $0.21,0.35 ; p=0.051$; Figure 1).

Each of the ROB domains and the overall ratings were examined in the context of the following variables: trial registration, industry funding, multi-centre status, number of treatment arms, intervention type, primary outcome category, and type of journal (see Additional file 3). Of these variables, trial registration had the most influence on ROB. Compared to trials that were not registered, those trials that were had a lower overall $\mathrm{ROB}$, as well as a lower ROB for each of the domains except selective outcome reporting. Odds ratios for high ROB ranged from 0.29 (95\% CI 0.12,0.69) for overall ROB to 0.47 (95\% CI $0.27,0.81$ ) for "other" sources of bias. Trials that were sponsored by industry were more likely to have adequate blinding than non-industry funded trials (OR 0.41 (95\% CI 0.22,0.76)), but were also more likely to be associated with "other" sources of bias (OR 4.72 (95\% CI 2.46,9.07)). ROB for selective outcome reporting increased with number of arms in the trial $(p=0.007)$, but was unchanged for the other domains. When compared to pharmacological interventions, trials investigating devices had a higher $\mathrm{ROB}$ associated with blinding (OR 3.37 (95\% CI 1.62,7.02)) and incomplete data (OR 2.56 (95\% CI 1.26,5.21)). High ROB due to blinding was also found in studies with outcomes related to techniques/training (e.g., longevity of dental restorations) when compared to physiological outcomes (OR 5.28 (95\% CI 1.09,25.61)). Multi-centre status and type of journal had no impact on ROB. 
Table 4 Trial characteristics and quality assessment stratified by trial registration and overall risk of bias ( $\mathbf{N}=300$ )

\begin{tabular}{|c|c|c|c|c|c|c|}
\hline Trial Characteristics & $\mathrm{N}$ & Trial Registered & & Risk of Bias & & \\
\hline & 300 & Yes $(\mathrm{N}=69 ; 23 \%)$ & $\begin{array}{l}\text { No } \\
\text { (N = 231;77\%) }\end{array}$ & $\begin{array}{l}\text { Low } \\
(\mathrm{N}=23 ; 8 \%)\end{array}$ & $\begin{array}{l}\text { Unclear } \\
(\mathrm{N}=99 ; 33 \%)\end{array}$ & $\begin{array}{l}\text { High } \\
(\mathrm{N}=178 ; 59 \%)\end{array}$ \\
\hline Impact factor (median, range) & 294 & $4.017(0.581-52.589)$ & $1.883(0.080-15.484)$ & $2.948(0.475-10.169)$ & $1.850(0.329-52.589)$ & $2.342(0.080-28.638)$ \\
\hline \multicolumn{7}{|l|}{ Continent of corresponding author } \\
\hline Africa & 11 & $4(5.8)$ & $7(3.0)$ & $1(4.4)$ & $4(4.0)$ & $6(3.4)$ \\
\hline Asia & 58 & $6(8.7)$ & $52(22.5)$ & $6(26.1)$ & $27(27.3)$ & $25(14.0)$ \\
\hline Australia & 16 & $1(1.5)$ & $15(6.5)$ & $2(8.7)$ & $7(7.1)$ & $7(3.9)$ \\
\hline Europe (excluding UK) & 91 & $17(24.6)$ & $74(32.0)$ & $6(26.1)$ & $27(27.3)$ & $58(32.6)$ \\
\hline North America & 87 & $30(43.5)$ & $57(24.7)$ & $5(21.7)$ & $19(19.2)$ & $63(35.4)$ \\
\hline South America & 7 & $2(2.9)$ & $5(2.2)$ & - & $2(2.0)$ & $5(2.8)$ \\
\hline United Kingdom & 30 & $9(13.0)$ & $21(9.1)$ & $3(13.0)$ & $13(13.1)$ & $14(7.9)$ \\
\hline Funding source specified & 194 & $62(89.9)$ & $132(57.1)$ & $23(100.0)$ & $49(49.5)$ & $122(68.5)$ \\
\hline Industry supported & 67 & $24 / 62(38.7)$ & 43/132 (32.6) & $7 / 23(30.4)$ & $8 / 49(16.3)$ & $52 / 122(42.6)$ \\
\hline Primary outcome explicitly stated & 123 & $41(59.4)$ & $82(35.5)$ & $14(60.9)$ & $34(34.3)$ & $75(42.1)$ \\
\hline Statistically significant outcome & 230 & $47(68.1)$ & $183(79.2)$ & $15(65.2)$ & $83(83.8)$ & $132(74.2)$ \\
\hline Data Monitoring Committee & 14 & $9(13.0)$ & $5(2.2)$ & $1(4.4)$ & $5(5.1)$ & $8(4.5)$ \\
\hline Jadad score (mean; median, range) & 300 & $2.99(3 ; 0-5)$ & $2.44(2 ; 0-5)$ & $3.96(4 ; 3-5)$ & $2.24(2 ; 1-5)$ & $2.56(2 ; 0-5)$ \\
\hline \multicolumn{7}{|l|}{ Allocation Concealment } \\
\hline Adequate & 65 & $24(34.8)$ & $41(17.8)$ & $20(87.0)$ & $15(15.2)$ & $30(16.9)$ \\
\hline Unclear & 227 & $45(65.2)$ & $182(78.8)$ & $3(13.0)$ & $84(84.9)$ & $140(78.7)$ \\
\hline Inadequate & 8 & - & $8(3.5)$ & - & - & $8(4.5)$ \\
\hline Trial registered & 69 & NA & NA & $11(47.8)$ & $10(10.1)$ & $48(27.0)$ \\
\hline \multicolumn{7}{|l|}{ Risk of Bias } \\
\hline Low & 23 & $11(15.9)$ & $12(5.2)$ & NA & NA & NA \\
\hline Unclear & 99 & $10(14.5)$ & 89 (38.5) & & & \\
\hline High & 178 & 48 (69.6) & $130(56.3)$ & & & \\
\hline
\end{tabular}

Trial registration was declared in the publication of 37 trials $(12.3 \%)$ and 69 records of registration $(23.0 \%)$ were found online. Registered trials were more likely to be published in journals with a higher impact factor (median $4.017 v s .1 .883 ; p<0.0001$ ). Approximately one third of trials were registered in studies with corresponding authors from Africa (36.4\%), North and South America (34.5\% and 28.6\%, respectively), and the UK (30.0\%), but proportions were lower for the rest of Europe (18.7\%), Asia (10.3\%), and Australia (6.3\%). Registered trials more often specified their funding source (89.9\% vs. 57.1\%; $p<0.0001$ ), and less often reported statistically significant findings, although this comparison was not statistically significant $(68.1 \%$ vs. $79.2 \% ; p=$

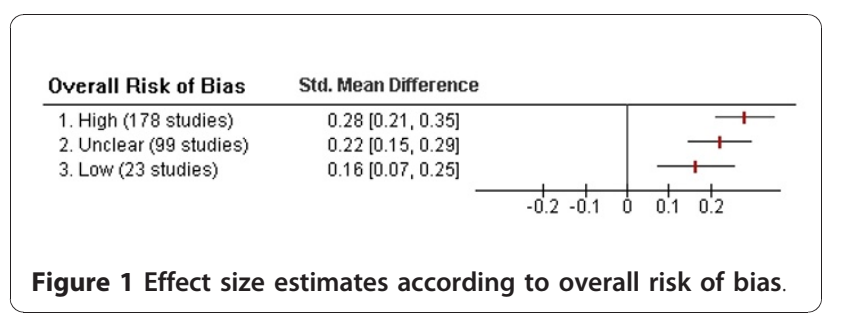

0.07). Measures of methodological quality were superior in registered trials (Table 4).

\section{Author Follow-Up Survey}

145 authors (50.0\%) responded to the survey, therefore the ability to generalize findings is limited. Of respondents, 61 (42.4\%) reported registration with a public trial registry, potentially corresponding closely to the 69 found in our search. The majority of these were registered with ClinicalTrials.gov (67.5\%) or Current Controlled Trials (17.5\%). 51.2\% were registered prior to and $37.2 \%$ after patient recruitment. The most common reason for registering a trial was a journal requirement for publication (72.7\%), followed by a belief in full public disclosure (68.2\%). For non-registration, the most common reasons were lack of familiarity with the process (59.1\%) and trial initiation prior to registration endorsement by the ICMJE (51.5\%) (Table 5).

Nearly all respondents (92.2\%) had prepared a study protocol prior to trial initiation; $2.0 \%$ reported a minor difference between the protocol and study conduct. $9.7 \%$ of authors reported that some outcomes measured in the trial were not reported in the publication. Space 
Table 5 Author responses to follow-up survey $(\mathrm{N}=145)$

\begin{tabular}{|c|c|}
\hline Survey Question & $\mathrm{N}(\%)$ \\
\hline \multicolumn{2}{|l|}{ Was your trial registered with a public trial registry? } \\
\hline Yes & $61(42.4)$ \\
\hline No & $83(57.6)$ \\
\hline No response & 1 \\
\hline \multicolumn{2}{|l|}{ What were your reasons for registering your trial (select all that apply)? } \\
\hline I believe that trials should be registered as a means of full public disclosure & $30(68.2)$ \\
\hline I endorse the statement regarding public trial registration made by the ICJME & $23(52.3)$ \\
\hline Trial registration is necessary for publication in some peer-reviewed journals & $32(72.7)$ \\
\hline Trial registration was required by the funding agency & $5(11.4)$ \\
\hline Trial registration was required by the Research Ethics Board & $9(20.5)$ \\
\hline Trial registration is institutional policy & $2(4.5)$ \\
\hline Other & $3(6.8)$ \\
\hline No response & 101 \\
\hline \multicolumn{2}{|l|}{ What were your reasons for not registering your trial (select all that apply)? } \\
\hline Lack of time & $3(4.5)$ \\
\hline Lack of resources & $5(7.6)$ \\
\hline I was not familiar with the process for trial registration & $39(59.1)$ \\
\hline Cost associated with registration & $4(6.1)$ \\
\hline I don't see a benefit to trial registration & $1(1.5)$ \\
\hline Trial was initiated prior to registration endorsement by the ICMJE & $34(51.5)$ \\
\hline No formal requirement & $4(6.1)$ \\
\hline Other & $7(10.6)$ \\
\hline No response & 79 \\
\hline
\end{tabular}

limitations were the most common concern (journal imposed space limitation 41.7\%; authors' concern about space $25.0 \%$ ), followed by non-statistically significant findings $(41.7 \%)$. While $56.4 \%$ of respondents indicated that they were willing to share their protocol, only two were received. In both cases, the details in the publication were consistent with the protocol.

\section{Discussion}

Our sample of recently-published pediatric trials demonstrates that there is considerable room for improvement in their design, conduct, and reporting. Methodological quality was modest, with the vast majority of trials at high or unclear ROB. Further, the trials did not adhere to widely accepted reporting standards or requirements for trial registration.

Our sample was intended to be representative of all RCTs published in 2007; therefore we placed no restrictions on journal, clinical area, or type of intervention. Trials in our sample were largely published in specialty journals, and examined a variety of interventions among a diverse range of conditions.

Methodological quality was assessed using three wellrecognized tools and the results overall were not favorable, suggesting methodological weaknesses and high risk of bias. Incomplete reporting was prevalent; while statements declaring implementation of certain design features (e.g., randomization and "double-blinding") were common, detailed methods were often not specified. Further, allocation concealment was rarely addressed at all. Despite the differing emphasis of the tools used (i.e., conduct for ROB and quality of reporting for Jadad and CONSORT), the results were consistent in that overall, the trials did not meet the criteria of any of the methods of assessment. However, there is evidence to suggest that the Jadad scale and $\mathrm{ROB}$ measure different constructs and that the assessment of ROB may be more appropriate [16].

Selective outcome reporting is of great concern. It is one of the driving forces for the promotion of trial registration and has important implications for safety [17-20]. To assess this domain, we compared the outcomes specified in the protocol or in the trial register to those reported in the publication; however the lack of registered trials and the extremely low response to requests for protocols made this difficult. As a result, our findings likely underestimate the risk associated with this particular issue, as we were unable to assess potential biases introduced through discrepancies between the original trial design and actual conduct.

Evidence suggests that industry-funded trials are more likely to report favorable results [21-23], therefore we included a criterion within the "other" sources of bias domain that related to inappropriate influence of the 
funding body. Provided that the source of funding was declared and a statement was made outlining the role of the sponsor, we considered the trial to be low ROB for that measure; however this information was often missing. While funding source was not the only consideration in assessing "other" sources of bias, it was relevant to every trial, and was therefore important in the determination of our overall results showing high or unclear ROB for this domain among two thirds of trials.

We found a noteworthy trend toward increasing effect estimates with increasing ROB which is consistent with previous research [16]. Trials at high ROB had a larger mean effect size than trials at low ROB, indicating the potential for a high proportion of trials to be reporting exaggerated results. These results are exploratory and should be interpreted with caution given the heterogeneity in outcomes compared and the small number of studies. Further work and methods that better account for confounding due to intervention and diagnostic condition are required before firm conclusions can be made.

Despite wide support [24], uptake by journals of the CONSORT Statement has been variable. In a survey of 165 high impact journals in 2007, 38\% mentioned the CONSORT Statement in the instructions to authors and $14 \%$ required (rather than recommended) it to be completed for a trial to be accepted [25]. This variability is echoed in our sample, as very few trials met all of the requirements of the checklist. Of the 11 trials that at least partially met all requirements, nine journals were represented. Of these, two journals stated in their instructions to authors that a completed CONSORT checklist was required, three recommended following the CONSORT guidelines, and the remainder did not mention the CONSORT Statement. Our observation that journal endorsement of the CONSORT Statement has little bearing on whether all of the recommended elements are reported highlights the practical issue of how to ensure adherence to the guidelines, and ultimately their impact on reporting.

Very few trials in our sample were registered in a public registry, and only about half of those that were registered declared this in the publication. Prospective trial registration has been heavily endorsed, and the volume of trials registered appears to be increasing [26-28]. However, trial registration is far from universal, and is perhaps more problematic in pediatric trials. Pandolfini and Bonati [29] found that the proportion of pediatric trials among all registered trials in online registers was disproportionate to those in the published literature. Pediatric trials are more likely to be published in specialty journals which may be less likely to require trial registration than general medical journals. Another concern is that the requirement for trial registration may not be enforced. Our author survey suggests that one of the major barriers to trial registration among respondents is a lack of familiarity with the process; therefore, efforts are required to raise awareness. These efforts should target researchers at the study design stage, rather than at the point of publication. However, reluctance on the part of academic researchers to publicly disclose the information required by trial registers may pose a challenge [30], an issue that was reinforced in this study by the apparent futility of contacting authors for access to protocol data. Potential future directions in this area may include the requirement of publicly available protocols at the time of trial registration or with funding applications.

Based on our findings, there is clearly room for improvement in pediatric trials. This is the mission of StaR Child Health (Standards for Research in Child Health), an international group that was recently formed involving varied stakeholders to develop and promote guidance to ensure the validity and relevance of pediatric trials [31]. With the involvement of trialists, clinicians, regulators, editors, and representatives of the pharmaceutical industry, this initiative is invested in meeting the needs of the research and clinical communities [32]. Through the development of standards for research in priority areas for pediatric research (e.g. appropriate outcome selection, data and safety monitoring committees, sample size, ROB), StaR Child Health aims to be an important contributor to a methodologically strong evidence base for pediatric care [33].

\section{Limitations}

We included approximately $10 \%$ of pediatric RCTs published in 2007, potentially limiting representativeness. Only trials published in English were included, possibly contributing to the high proportion of studies from North America and the UK. While we extracted the country of the corresponding author, this is not a perfect proxy for the population studied and in some cases, an author from a high income country reported on a trial conducted in a low or middle income area.

The true ROB was often difficult to interpret in our sample due to poor reporting. Additionally, the issue of selective outcome reporting posed a challenge as protocols or trial registers were unavailable for the majority of studies. In most cases, the publication was judged according to its internal consistency. Hence, the high proportion of trials that were given a rating of low $\mathrm{ROB}$ for this domain likely underestimates the true ROB.

The pooled analysis presented to examine trends in effect sizes and ROB is preliminary work. Given the heterogeneity in diseases, interventions, and outcomes included in the sample, we used standardized measures of effect size to be able to investigate general patterns across studies, but these results are exploratory. 
The response to our author survey was likely subject to response bias. The item responses indicate that authors who had registered their trials were more likely to participate in the survey, potentially limiting applicability. Assuming that respondents were more aware of issues related to trial registration and methodological initiatives in general, the answers provided (for example reasons for non-registration) may not encompass some of the deeper issues faced by other researchers and may have implications for attempts to overcome these barriers in the future.

\section{Conclusions}

This study shows that the majority of pediatric trials published in 2007 were at high risk of bias, corresponding with a trend toward increased effect sizes. In spite of a movement towards improving methodological quality and requirements for trial registration, the majority of trials have not met these recommendations. These results should be of great concern for child health providers, researchers, methodologists, and funders, and should motivate all to work towards improving the design, conduct, and reporting of child health research.

\section{Additional material}

\section{Additional file 1: Guidelines and Decision Rules for Risk of Bias}

Assessments. List of decision rules developed by our research group to be used with the Cochrane Handbook in assessing risk of bias.

Additional file 2: Author Follow-Up Survey. Included questions in the survey sent to 290 corresponding authors.

Additional file 3: Odds Ratios for Risk of Bias by Selected Variables. Odds ratios for high risk of bias by selected variables, stratified by the six domains of the Cochrane Collaboration's Risk of Bias tool.

\section{Acknowledgements}

We thank Ahmed Abou-Setta, Liza Bialy, Jeff Klassen, Shima Mousavi, Kate O'Gorman, Janine Schouten, and Kai Wong for their assistance in completing the Risk of Bias assessments. This study was partially funded by the Women \& Children's Health Research Institute. The funders had no role in study design, data collection and analysis, decision to publish, or preparation of the manuscript.

\section{Author details}

${ }^{1}$ Alberta Research Centre for Health Evidence, Department of Pediatrics, University of Alberta. Edmonton, Canada. ${ }^{2}$ Division of Pediatric Emergency Medicine, Department of Pediatrics, University of Alberta. Edmonton, Canada. ${ }^{3}$ Women and Children's Health Research Institute, University of Alberta. Edmonton, Canada. ${ }^{4}$ Manitoba Institute of Child Health, Winnipeg, Canada.

\section{Authors' contributions}

$\mathrm{MPH}$ contributed to study design, coordinated the study, extracted data, performed quality and risk of bias assessments, analyzed data, and drafted the manuscript. LH contributed to study design, provided oversight for the study and analysis, contributed to interpretation of data, helped draft and edit the manuscript, and is guarantor. AM extracted data, conducted quality and risk of bias assessments, and critically reviewed the manuscript. LT performed the literature search. BV performed statistical analysis and interpreted the data. DT and SC contributed to study design and critically reviewed the manuscript. TPK contributed to study design, provided oversight for the study, and critically reviewed the manuscript. All authors gave final approval of the version to be published.

\section{Competing interests}

The authors declare that they have no competing interests.

Received: 31 August 2010 Accepted: 22 December 2010 Published: 22 December 2010

\section{References}

1. Cohen $E$, Uleryk E, Jasuja M, Parkin PC: An absence of pediatric randomised controlled trials in general medical journals, 1985-2004. J Clin Epidemiol 2007, 60:118-123.

2. Martinez-Castaldi C, Silverstein M, Bauchner $\mathrm{H}$ : Child versus adult research: the gap in high-quality study design. Pediatrics 2008, 122:52-57.

3. Higgins JPT, Green S, eds: Cochrane handbook for systematic reviews of interventions version 5.0.0 Cochrane Collaboration; 2008.

4. Jadad AR, Moore RA, Carroll D, Jenkinson C, Reynolds DJM, Gavaghan DJ, McQuay HJ: Assessing the quality of reports of randomized clinical trials: is blinding necessary? Control Clin Trials 1996, 17:1-12.

5. Schulz KF, Chalmers I, Hayes RJ, Altman DG: Empirical evidence of bias. Dimensions of methodological quality associated with estimates of treatment effects in controlled trials. JAMA 1995, 273:408-412.

6. DeAngelis CD, Drazen JM, Frizelle FA, Haug C, Hoey J, Horton R, Kotzin S, Laine C, Marusic A, Overbeke AJ, Schroeder TV, Sox HC, Van Der Weyden MB: Clinical trial registration: a statement from the International Committee of Medical Journal Editors. JAMA 2004, 292:1363-1364.

7. Schulz KF, Altman DG, Moher D, for the CONSORT Group: CONSORT 2010 Statement: updated guidelines for reporting parallel group randomised trials. BMJ 2010, 340:C332.

8. DeAngelis CD, Drazen JM, Frizelle FA, Haug C, Hoey J, Horton R, Kotzin S, Laine C, Marusic A, Overbeke AJ, Schroeder TV, Sox HC, Van Der Weyden MB: Is this clinical trial fully registered?: a statement from the International Committee of Medical Journal Editors. JAMA 2005, 293:2927-2929.

9. Mathieu S, Boutron I, Moher D, Altman DG, Ravaud P: Comparison of registered and published primary outcomes in randomised controlled trials. JAMA 2009, 302:977-984.

10. Plint AC, Moher D, Morrison A, Schulz K, Altman DG, Hill C, Gaboury I: Does the CONSORT checklist improve the quality of reports of randomised controlled trials? A systematic review. MJA 2006, 185:263-267.

11. Hopewell S, Dutton S, Yu LM, Chan AW, Altman DG: The quality of reports of randomised trials in 2000 and 2006: comparative study of articles indexed in PubMed. BMJ 2010, 340:c723.

12. Boluyt N, Tjosvold L, Lefebvre C, Klassen TP, Offringa M: The usefulness of systematic review search strategies in finding child health systematic reviews in MEDLINE. Arch Ped Adolesc Med 2008, 162:111-116.

13. Thomson D, Hartling L, Cohen E, Vandermeer B, Tjosvold L, Klassen TP: Controlled trials in children: Quantity, methodological quality and descriptive characteristics of pediatric controlled trials published 19482006. Plos One 2010, 5(9):e13106.

14. Altman DG, Schulz KF, Moher D, Egger M, Davidoff F, Elbourne D, Gøtzsche PC, Lang T, CONSORT Group: The revised CONSORT Statement for reporting randomized trials: explanation and elaboration. Ann Intern Med 2001, 134:663-694.

15. Borenstein M, Hedges LV, Higgins JPT, Rothstein HR: Converting Among Effect Sizes. In Introduction to Meta-Analysis. Volume Chapter 6. John Wiley and Sons, Ltd, Chichester, UK; 2009:45-49.

16. Hartling L, Ospina M, Liang Y, Dryden DM, Hooton N, Krebs Seida J, Klassen TP: Risk of bias versus quality assessment of randomised controlled trials: cross sectional study. BMJ 2009, 339:b4012.

17. Chan AW, Hróbjartsson A, Haahr MT, Gøtzsche PC, Altman DG: Empirical evidence for selective reporting of outcomes in randomized trials: Comparison of protocols to published articles. JAMA 2004, 291:2457-2465.

18. Chan AW, Krleža-Jerić K, Schmid I, Altman DG: Outcome reporting bias in randomized trials funded by the Canadian Institutes of Health Research. CMAJ 2004, 171:735-740.

19. Dwan K, Altman DG, Arnaiz J, Bloom J, Chan AW, Cronin E, Decullier E, Easterbrook PJ, Von Elm E, Gamble C, Ghersi D, loannidis JP, Simes J, 
Williamson PR: Systematic review of the empirical evidence of study publication bias and outcome reporting bias. PLOS One 3(8):e3081.

20. Benjamin DK, Smith PB, Sun JM, Murphy MD, Avant D, Mathis L, Rodriguez W, Califf RM, Li JS: Safety and transparency of pediatric drug trials. Arch Pediatr Adolesc Med 2009, 163:1080-1086.

21. Bekelman JE, Li Y, Gross CP: Scope and impact of financial conflicts of interest in biomedical research: a systematic review. JAMA 2003, 289:454-469.

22. Lexchin J, Bero LA, Djulbegovic B, Clark O: Pharmaceutical industry sponsorship and research outcome and quality: systematic review. BMJ 2003, 326:1167-1170.

23. Sismondo S: Pharmaceutical company funding and its consequences: a qualitative systematic review. Cont Clin Trials 2008, 29:109-113.

24. Altman DG: Endorsement of the CONSORT statement by high impact medical journals: survey of instructions for authors. BMJ 2005, 330:1056-1057.

25. Hopewell S, Altman DG, Moher D, Schulz KF: Endorsement of the CONSORT Statement by high impact factor medical journals: a survey of journal editors and journal 'Instructions to Authors'. Trials 2008, 9:20.

26. Laine C, Horton R, DeAngelis CD, Drazen JM, Frizelle FA, Godlee F, Haug C, Hébert PC, Kotzin S, Marusić A, Sahni P, Schroeder TV, Sox HC, Van Der Weyden MB, Verheugt FW: Clinical trial registration - looking back and moving ahead. JAMA 2007, 298:93-94.

27. Rennie D: Trial registration: a great idea switches from ignored to irresistible. JAMA 2004, 292:1359-1362.

28. Zarin DA, Ide NC, Tse T, Harlan WR, West JC, Lindberg DA: Issues in the registration of clinical trials. JAMA 2007, 297:2112-2120.

29. Pandolfini C, Bonati M: Children's presence in research. A review of online registers. Eur J Clin Pharmacol 2009, 65(9):873-80.

30. Scherer $M$, Trelle $S$ : Opinions on registering trial details: a survey of academic researchers. BMC Health Serv Res 2008, 8:18.

31. Klassen TP, Hartling L, Hamm M, van der Lee JH, Ursum J, Offringa M: StaR Child Health: an initiative for RCTs in children. Lancet 2009, 374:1310-1312.

32. Veitch E: StaRs of child health. 2009 [http://speakingofmedicine.plos.org/ 2009/11/05/stars-of-child-health/, Accessed 11 February 2010.

33. Klassen TP, Hartling L, Craig JC, Offringa M: Children are not just small adults: the urgent need for high-quality trial evidence in children. PLOS Med 2008, 5:e172.

\section{Pre-publication history}

The pre-publication history for this paper can be accessed here: http://www.biomedcentral.com/1471-2431/10/96/prepub

doi:10.1186/1471-2431-10-96

Cite this article as: Hamm et al: A descriptive analysis of a representative sample of pediatric randomized controlled trials published in 2007. BMC Pediatrics 2010 10:96.

\section{Submit your next manuscript to BioMed Central and take full advantage of:}

- Convenient online submission

- Thorough peer review

- No space constraints or color figure charges

- Immediate publication on acceptance

- Inclusion in PubMed, CAS, Scopus and Google Scholar

- Research which is freely available for redistribution

Submit your manuscript at www.biomedcentral.com/submit
Biomed Central 